\title{
Meloxicam ameliorates the cartilage and subchondral bone deterioration in monoiodoacetate-induced rat osteoarthritis
}

\author{
Előd Nagy ${ }^{\text {Corresp.. }}{ }^{1}$ ， Enikő Vajda ${ }^{2}$, Camil Vari ${ }^{3}$ ， Sándor Sipka ${ }^{4}$ ，Ana-Maria Fárr ${ }^{5}$ ， Emőke Horváth ${ }^{6}$ \\ ${ }^{1}$ Dept. of Biochemistry and Environmental Chemistry, University of Medicine and Pharmacy, Targu-Mures,, Romania \\ 2 Dept. of Drug Analysis, University of Medicine and Pharmacy, Targu-Mures, Romania \\ 3 Dept. of Pharmacology, University of Medicine and Pharmacy of Targu Mures, Targu-Mures, Romania \\ 4 Department of Clinical Immunology, University Medical School of Debrecen, Debrecen, Hungary \\ 5 Dept. of Pathophysiology, University of Medicine and Pharmacy of Targu Mures, Targu-Mures, Romania \\ 6 Dept. of Pathology, University of Medicine and Pharmacy of Targu Mures, Targu-Mures, Romania \\ Corresponding Author: Elöd Nagy \\ Email address: elod.nagy@umftgm.ro
}

Objective. This study aimed to quantify the cartilage- and subchondral bone-related effects of low-dose and high-dose meloxicam treatment in the late phase of monoiodoacetate-induced osteoarthritis of the stifle. Methods. Thirty-four male Wistar rats received intra-articular injection of mono-iodoacetate to trigger osteoarthritis; 10 control animals (Grp Co) received saline. The mono-iodoacetate-injected rats were assigned to 3 groups and treated from week 4 to the end of week 7 with placebo (Grp P, n=11), low-dose (GrpM Lo, $0.2 \mathrm{mg} / \mathrm{kg}, \mathrm{n}=12$ ) or high-dose (GrpM Hi, $1 \mathrm{mg} / \mathrm{kg}, \mathrm{n}=11$ ) meloxicam. After a period of 4 additional weeks (end of week 11 ) the animals were sacrificed, and the stifle joints were examined histologically and immunohistochemically for cyclooxygenase 2 , in conformity with recommendations of the Osteoarthritis Research Society International. Serum cytokines IL-6, TNF $\alpha$ and IL-10 were measured at the end of weeks 3, 7, and 11 . Results. Compared with saline-treated controls, animals treated with mono-iodoacetate developed various degrees of osteoarthritis. The cartilage degeneration score and the total cartilage degeneration width were significantly lower in both the low-dose $(p=0.012$ and $p=0.014)$ and high-dose $(p=0.003$ and $p=0.006)$ meloxicam-treated groups than in the placebo group. In the subchondral bone, only high-dose meloxicam exerted a significant protective effect $(p=0.011)$. Low-grade Cox-2 expression observed in placebo-treated animals was abolished in both meloxicam groups. Increase with borderline significance of TNF $\alpha$ in GrpP from week 3 to week $7(p=0.049)$ and reduction of IL- 6 in GrpM Lo from week 3 to week $11(p=0.044)$ were observed. Conclusion. In this rat model of osteoarthritis, both low-dose and high-dose meloxicam had a chondroprotective effect, and the high dose also protected against subchondral bone lesions. The results suggest a superior protection of the high-dose meloxicam arresting the low-grade inflammatory pathway accompanied 
by chronic cartilage deterioration. 
1

2

3

4

5

6 1- Dept. of Biochemistry and Environmental Chemistry, University of Medicine and Pharmacy,

7 Targu-Mures, Romania

8 2- Dept. of Drug Analysis, University of Medicine and Pharmacy, Targu-Mures, Romania

9 3- Dept. of Pharmacology, University of Medicine and Pharmacy, Targu-Mures, Romania

10 4- Department of Clinical Immunology, University of Debrecen, Hungary

11 5- Dept. of Pathophysiology, University of Medicine and Pharmacy, Targu-Mures, Romania

12 6- Dept. of Pathology, University of Medicine and Pharmacy, Targu-Mures, Romania

\section{3}

14

15

16

17 *corresponding author: Előd Ernő Nagy, Dept. of Pharmaceutical Biochemistry, University of Medicine and Pharmacy Târgu-Mureș, Gh. Marinescu str. 38., Târgu-Mureș 540139, Romania e-mail: elod.nagy@umftgm.ro 

was published: Nagy, E. et al, Standardized histological assessment of late-phase meloxicam

21 effect in iodoacetate-induced rat osteoarthritis. In Osteoporosis International, Volume 27, 22 Supplement 1, April 2016. Springer London Ltd., London, England.

\section{ABSTRACT}

Objective. This study aimed to quantify the cartilage- and subchondral bone-related effects of low-dose and high-dose meloxicam treatment in the late phase of mono-iodoacetateinduced osteoarthritis of the stifle.

Methods. Thirty-four male Wistar rats received intra-articular injection of mono29 iodoacetate to trigger osteoarthritis; 10 control animals (Grp Co) received saline.

The mono-iodoacetate-injected rats were assigned to 3 groups and treated from week 4 to

31 the end of week 7 with placebo (Grp P, n=11), low-dose (GrpM Lo, $0.2 \mathrm{mg} / \mathrm{kg}, \mathrm{n}=12$ ) or highdose (GrpM Hi, $1 \mathrm{mg} / \mathrm{kg}, \mathrm{n}=11)$ meloxicam. After a period of 4 additional weeks (end of week 11) the animals were sacrificed, and the stifle joints were examined histologically and immunohistochemically for cyclooxygenase 2 , in conformity with recommendations of the measured at the end of weeks 3,7 , and 11 .

Results. Compared with saline-treated controls, animals treated with mono-iodoacetate developed various degrees of osteoarthritis. The cartilage degeneration score and the total cartilage degeneration width were significantly lower in both the low-dose $(p=0.012$ and 
$40 \mathrm{p}=0.014)$ and high-dose $(\mathrm{p}=0.003$ and $\mathrm{p}=0.006)$ meloxicam-treated groups than in the placebo

41 group. In the subchondral bone, only high-dose meloxicam exerted a significant protective

42 effect $(p=0.011)$. Low-grade Cox-2 expression observed in placebo-treated animals was

43 abolished in both meloxicam groups. Increase with borderline significance of TNF $\alpha$ in GrpP

44 from week 3 to week $7(p=0.049)$ and reduction of IL-6 in GrpM Lo from week 3 to week 11

$45(\mathrm{p}=0.044)$ were observed.

46 Conclusion. In this rat model of osteoarthritis, both low-dose and high-dose meloxicam

47 had a chondroprotective effect, and the high dose also protected against subchondral bone

48 lesions. The results suggest a superior protection of the high-dose meloxicam arresting the low-

49 grade inflammatory pathway accompanied by chronic cartilage deterioration. 


\section{INTRODUCTION}

Osteoarthritis (OA) is a complex chronic disorder characterized by loss and metabolic

56

changes of the cartilage matrix along with low-grade inflammation and alterations of the subchondral bone. The degradative pathways were initially thought to be cartilage-driven, but newer evidence has documented that they are substantially influenced by inflammatory mediators released from the subchondral bone (Westacott et al., 1997; Berenbaum, 2013; Yu et al., 2016). An up-regulated signalling cross-talk between the osteoarthritic subchondral bone and cartilage has been revealed (Yuan et al., 2014). In experimental osteoarthritis of the stifle, intraarticular injection of mono-iodoacetate (MIA), a glyceraldehide-3-phosphate dehydrogenase inhibitor, halts the glycolysis, provoking chondrocyte death (especially in the central tibial zone), loss of proteoglycans, fibrillation, and formation of cysts and large osteophytes (Bendele, 2001). The chronologic development of histological lesions in MIA-induced osteoarthritis has been described in detail (Guzman et al., 2003). The predominant early symptoms of MIA toxicity are shrinking, degeneration and death of chondrocytes, with synovial edema and a moderate mononuclear cell infiltrate (Guzman et al., 2003). After a week, clustered osteoblasts and increased osteoclast activation can be observed, indicating increased bone remodelling. Cartilage fragmentation and erosion in tight anatomic relationship with collapse and fragmentation of the underlying bony trabeculae are present (Guzman et al., 2003). These histological changes occur on the background of variations in biphasic proteoglycan synthesis (Dumond et al., 2004).

After MIA injection, cyclooxygenase-2 (Cox-2), together with matrix metalloproteinase-2 activity, is quickly up-regulated. Some important pro-inflammatory genes, such as interleukin-1 $\beta$ (IL-1 $\beta$ ), and inducible nitric oxide synthase (iNOS), also had increased activity in a MIA-model, 
77 in parallel with the down-regulation of proteoglycan synthesis in the tibial plateau and condyle

78 (Dumond et al., 2004). Experimental, but also human osteoarthritis are characterized by a self-

79 perpetuating, low-grade inflammation affecting both the synovial membrane and the cartilage.

80 Regulatory molecules, such as IL-1 $\beta$, leptin, TNF $\alpha$, receptor for advanced glycation end-

81 products (RAGE), and iNOS, confer a destruction-prone activated phenotype to chondrocytes

82 (Musumeci et al., 2015).

83 Orally administered Cox-2 selective non-steroidal anti-inflammatory agents may decrease

84 persistent low-grade inflammation. These drugs are recommended in the recent Osteoarthritis

85 Research Society International (OARSI) therapeutic guidelines for the treatment of knee-only

86 osteoarthritis in patients who have no co-morbidities or multi-joint osteoarthritis (McAlindon et.,

87 al 2015).

However, there is a need for more group-specific therapies, as orally administered meloxicam may be associated with serious dose-related adverse effects and lower-dose pharmaceutical formulations are under evaluation in phase 3 clinical studies (Altman et al.,

91 2015). As an alternative, experimental approaches with intra-articular repeated high doses of the

92 drug proved to be successful (Wen et al., 2013).

The grading system elaborated by Pritzker et al. proved to offer simplicity, scalability,

94 extendability and comparability to the histopathology evaluation (Custers et al., 2007; Pritzker et

95 al., 2006). In experimental conditions, detailed recommendations for the histopathological

96 examination of joint lesions of the rat were elaborated through the OARSI histopathology

97 initiative (Gerwin et al., 2010) in order to allow standardization and a better comparison of

98 various results. 
Since the histological changes in MIA-triggered osteoarthritis are progressive and the degenerative process in the subchondral bone peaks late, from day 42 to 56 (Guzman et al.,

101 2003; Miyamoto et al., 2016), we considered that this period corresponds to the late, chronic

102 phase of osteoarthritis. Further, this time period corresponds with the estimation that 11 weeks of

103 rat age equals several years in humans (Sengupta, 2013). We have hypothesized that low-grade

104 inflammation induced by MIA persists in the subchondral bone and bone marrow in the late-

105 phase reaction of osteoarthritis, thus sustaining cartilage destruction. We proposed that

106 meloxicam can ameliorate not only the cartilage deterioration, but also the lesions provoked on

107 subchondral bone. Our goal was to quantify the dose-related effects of meloxicam treatment in

108 the subchondral bone-involving, late phase of mono-iodoacetate-induced osteoarthritis, and to

109 follow the levels of serum IL-6, IL-10 and TNF $\alpha$, as markers of systemic inflammation.

110 In order to elucidate whether a daily single dose of meloxicam will maintain circulating

111 levels, and to establish the time to reach the steady-state plasma concentration, we performed a

112 pharmacokinetic pre-study of meloxicam.

Experimental design

METHODS

117 guidelines (Kilkenny et al., 2010). The major phases, grouping, timing of interventions, and

118 checkpoints of the experiment are summarized in a flow-chart below. (Figure 1.) 

and Pharmacy Targu-Mures (no.132/23.12.2013). The sample size was calculated by simulations assuming different means and slightly different variability of the two major histological scores

124 and IL-6 levels between the groups, setting $\alpha=0.05$ and $1-\beta 0.8$. Initially, 46 healthy adult male

125 Wistar rats (age: 6 months, body weight: 170-230 g), obtained from the University Biobase, were entered into the study. The animals were kept under controlled conditions (temperature $22.5 \pm$ $2^{\circ} \mathrm{C}$, humidity $55 \pm 5 \%$ ) on a 12-h light-dark cycle, 5-6 animals per cage (solid bottom), and fed with standard rat chow and water ad libitum. All procedures and experimental protocols used in this study were in compliance with the 2010/63/EU Directive of the European Parliament and

130 Council accepted at September 22, 2010, for the protection of animals used for scientific 131 purposes.

\section{The osteoarthritis model}

Forty-six animals were assigned to one of 4 groups by a simple randomization. The first group (GrpCo), consisting of 10 animals, was assigned to control conditions; animals in this group received only $50 \mu \mathrm{l}$ of intra-articular saline. The other 36 animals received sodium monoiodoacetate (MIA; Biochemica, Applichem Gmbh, Germany), 4 mg dissolved in $50 \mu 1$ of

137 physiological saline, injected into the joint cavity through the infrapatellar ligament of the left

138 stifle, with a 26-gauge, 0.5 inch needle. The intra-articular injection was performed under

139 anaesthesia with ketamine $10 \%(80 \mathrm{mg} / \mathrm{kg})$ plus xylazine $1 \%(5 \mathrm{mg} / \mathrm{kg})$. After MIA injection,

140 animals were carefully inspected daily by trained personnel blinded to treatment data to assess

141 stifle-joint swelling and dysfunction. 
osteoarthritis in each animal was confirmed by stifle radiography performed by a veterinarian low grades and no clear radiographic signs of osteoarthritis were excluded from the study.

GrpP $(n=11)$ received placebo solution (polyethylene glycol 400/water solution 50:50) chosen considering the human equivalent dose $(\mathrm{HED}=0.162 \times 1 \mathrm{mg} / \mathrm{kg})(\mathrm{Nair} \& \mathrm{Jacob}, 2016)$, while the low-dose was determined taking into consideration toxicological data indicating 0.2 $\mathrm{mg} / \mathrm{kg}$ as the "No Effect Level" on the gastrointestinal system and the kidney (Summary Report,

155 Committee for Veterinary Medicinal Products, The European Agency for the Evaluation of

156 Medicinal Products, Meloxicam, June 1997). The drug was dissolved in a polyethylene glycol 157 400/water solution (50:50) vehicle and administered daily by gavage, in a single dose between 9 158 and 10 a.m. for period of 4 weeks.

\section{Pharmacokinetic study of meloxicam}


163 hrs after the drug delivery in heparinized tubes. Plasma was separated by centrifugation at 3500 $164 \mathrm{rpm}, 10 \mathrm{~min}$. samples were diluted 1:10 with blank pooled plasma, internal standard was added, and

167 deproteinizing solution was applied. The diluted sample/internal standard/acetonitril/ $/ \mathrm{HClO}_{4}$

168 mixture was prepared in a proportion of 16:4:10:3, then vortexed for $15 \mathrm{sec}$, allowed to stand for

$1695 \mathrm{~min}$, and centrifuged $10 \mathrm{~min}$ at $10000 \mathrm{rpm}$. The supernate was analyzed on a Waters Symmetry

170 C-8 chromatography column, 4.6 x $150 \mathrm{~mm}, 5 \mu \mathrm{m}$ with a Merck-Hitachi-LaChema instrument.

171 The elution buffer was $1 \%$ aqueous solution of acetic acid:acetonitril (60:40), yield $1.7 \mathrm{~mL} / \mathrm{min}$;

172 detection was performed by diode array detector (DAD) at $355 \mathrm{~nm}$.

173 The pharmacokinetic parameters were determined by use of the software Kinetica 5.1

174 SP1 (Thermo Fisher Scientific Inc.). The AUC was calculated with a mixed log-linear method, 175 and all of the pharmacokinetic parameters were calculated by applying a non-compartmental 176 model.

\section{Blood sampling}

Baseline blood sampling was performed 3 weeks after the MIA injection, before start of meloxicam treatment. A second blood sampling took place at the end of meloxicam treatment, and the last one after 4 weeks of rest. Each time, $150 \mu \mathrm{L}$ of venous blood was drawn from the tail vein and centrifuged at $3500 \mathrm{rpm}$ for 10 minutes. Serum aliquots were stored at $-20{ }^{\circ} \mathrm{C}$ until

182 the determination of IL-6, Il-10 and TNF $\alpha$. 


\section{Serum cytokine measurement}

The thawed serum aliquots were used for the measurement of IL-6, TNF $\alpha$, and IL-10,

186 which was performed on a Luminex 200 platform, applying Fluorokine Map Rat IL-6 (LUR506),

187 IL-10 (LUR522), and TNFa/TNFSF2 (LUR510) kits (R\&D Systems, US).

\section{Histological analysis of the joints}

189

At the end-point, 4 weeks after meloxicam treatment was terminated, all animals were sacrificed by excessive halothane inhalation, realized with an anaesthetic vaporizer (halothane

191 saturation $6 \%, \mathrm{O}_{2} 1.5 \mathrm{~L} / \mathrm{min}$ ), followed by cervical dislocation. After absence of vital functions

192 had been determined by the veterinarian, osteoarticular samples were obtained from each animal

193 from the injected- and the contralateral side (limb). After soft tissue was removed, the remaining

194 material was fixed with formalin (4\% neutral-solution) for 3 days and decalcified in Richard-

195 Allan Scientific Decalcifying solution (Thermo Scientific, Kalamazoo, USA) for 2 days. The

196 specimens were processed by the frontal sectioning method followed by paraffin embedding

197 (Gerwin et al., 2010). From each sample, serial 4- $\mu \mathrm{m}$ sections were cut in about 300- $\mu \mathrm{m}$ steps,

198 followed by haematoxylin-eosin (HE) and periodic-acid-Schiff (PAS) staining. Microscopic

199 examination was performed by two independent investigators, blinded for the group

200 classification, with synchronous imaging on a trinocular microscope. The contralateral limb in

201 every case was investigated as a negative control, with a single section per each healthy joint.

202 On each section, four scores proposed by the OARSI histopathology initiative were

203 determined: the cartilage degeneration score (CDS), the total cartilage degeneration width

204 (TCDW), the calcified cartilage and subchondral bone damage score (SBD), and the synovial

205 reaction (SR) (Gerwin et al., 2010). The average values obtained from the two investigators were 
206

207

208

used for statistical analysis. Inter-observer Spearman correlation coefficients for these

parameters were the following: $r($ grade $)=0.97, r($ stage $)=0.90, r(\mathrm{CDS})=0.96, \mathrm{r}(\mathrm{TCDW})=0.98$, $\mathrm{r}(\mathrm{CCSBD})=0.94, \mathrm{r}(\mathrm{SR})=1$.

\section{Cox-2 immunohistochemistry}

Cox-2 was stained on a single section from each animal by applying a monoclonal antiCox2 antibody (SP21, Thermo Fisher Scientific) in a dilution of 1:100, with high $\mathrm{pH}$ retrieval solution, in conformity with the heat-induced epitope retrieval method, followed by 60 -min incubation at room temperature. The reaction product was developed with the reagent EnvisionFLEX High pH (Dako, Denmark) and made visible by reaction with diamino-benzidine. The

percentage of Cox-2 positive cells (chondrocytes in cartilage/fibroblasts, fibrocytes in subchondral bone, and hematopoietic elements in bone marrow) was separately assessed in 3 joint regions: cartilage, subchondral bone, and subchondral bone marrow.

\section{Statistical analysis}

The statistical analysis of data was performed with GraphPad Prism 7.01 (GraphPAd Software Inc., USA) and STATISTICA 5.0 (Statsoft, USA). The distribution of histological scores was analysed by use of the Lilliefors and the Shapiro-Wilk test and showed abnormality without exception; therefore, we performed non-parametric tests in interpretation: the KruskalWallis ANOVA test for multiple and the Mann-Whitney U test (two-tailed) for between-group comparison, the Spearman rank correlation for correlation analysis and the Wilcoxon matched pairs test for matched data series (logarithmically transformed cytokine levels). The threshold of significance was set to $\mathrm{p}=0.05$, and for each test we also calculated the effect size $(\mathrm{z}, \mathrm{r}$ in the Mann-Whitney U test, $\mathrm{r}$ in Spearman correlation). We applied Holm's sequential Bonferroni 
228 adjustment to the $p$ values obtained in multiple comparisons of the osteoarthritic groups (GrpP,

229 GrpM Lo, GrpM Hi).

230

231

RESULTS

232

Pharmacokinetic study of meloxicam

233

In the pharmacokinetic pre-study of meloxicam, the following characteristics were

234 recorded: $\mathrm{Cmax}=4.79 \pm 0.53 \mu \mathrm{g} / \mathrm{mL}, \mathrm{tmax}=8 \pm 3.1 \mathrm{hrs}, \mathrm{t}_{1 / 2}=15.38 \pm 2.91 \mathrm{hrs}, \mathrm{MRT}=25.61 \pm$

$2352.92 \mathrm{hrs}, \mathrm{AUC} \mathrm{t} \rightarrow \infty<13.5 \pm 4.1 \%$.

\section{Characteristic joint lesions of the groups}

There were minimal detectable lesions in specimens of GrpCo. In GrpP, tissue lesions

238

239

240

241

242

243

were the most severe, with the following key features: erosion, superficial delamination,

excavation with matrix loss extending to the mid zone, and denudation with a variable mass of fibrocartilaginous tissue, microfractures of the bone plate. Deformed fibrocartilaginous articular surface was present in 1 of 11 animals, and severe fragmentation of the calcified cartilage was present in 2 of 12. Significant (up to $3 / 4$ ) fibroblastic transformation of the subjacent bone marrow was present in 5 animals. (Figure 2.a-c.)

In comparison with these results, at GrpM Lo, the tissue lesions were reduced: half of the cases $(6 / 12)$ had moderate matrix loss with focal rarefaction and condensation of collagen fibers, mild-to-moderate grade of chondrocyte loss and superficial fissures. 4/12 cases presented deep fissures, a more intense matrix loss and collagen fiber condensation, and at 2/12 had denudation with complete erosion. Marked fragmentation of the calcified cartilage could be identified in 
$2492 / 12$ subjects, while important fibroblastic transformation of the bone marrow was seen also in

$250 \quad$ 2/12 specimens. (Figure 2.d-f.)

251

In $\mathrm{GrpM} \mathrm{Hi}$, even lesser pronounced histological deteriorations were observed. At 6/11

252 animals the most prominent changes were superficial matrix discontinuity with a low-grade

253 chondrocyte death or hypertrophy, 4/12 animals showed variable vertical fissures, and only 1/12

254 presented erosion, important subchondral fragmentation, and fibroblastic transformation. (Figure 255 2.g-i)

\section{The OARSI histopathology initiative scores}

All three osteoarthritic groups had significantly higher CDS, TCDW, SBD and SR scores

than did the GrpCo (data shown in Figure 3.). Multiple comparisons by the use of Kruskal-

Wallis ANOVA of GrpP, GrpM Lo and GrpM Hi showed a significant effect on CDS ( $\mathrm{p}=0.003$ ),

TCDW ( $\mathrm{p}=0.005), \operatorname{SBD}(\mathrm{p}=0.041)$. In GrpP, CDS was the highest possible (15) in 4 of 11

261

animals. In GrpM Lo, the range of the CDS was 3-12; in GrpM Hi, it was 5 to 11.

The histological scores of the study groups, are shown on Figure 3 and in the

Supplementary Table. CDS was significantly lower in GrpM Lo and GrpM Hi than in GrpP: 8.75

(6.5-10.5) and $8(6-10.5) v s .12(11-15), \mathrm{p}=0.012(\mathrm{z}=2.67, \mathrm{r}=0.56)$ and $\mathrm{p}=0.003,(\mathrm{z}=3.08, \mathrm{r}=0.66)$.

Similarly, the TCDW was significantly lower in GrpM Lo and GrpM Hi than in GrpP: 875 (600-

$\mathrm{p}=0.006(\mathrm{z}=2.99, \mathrm{r}=0.64)$. The SBD score was significantly lower in GrpM Hi than in GrpP- 1 $1(0.75-2.5) v s .2 .5(2-3), \mathrm{p}=0.10(\mathrm{z}=1.63, \mathrm{r}=0.34) . \mathrm{CDS}, \mathrm{TCDW}$ and SBD scores of GrpM Lo 
270 and GrpM Hi were not significantly different. Synovial reaction (SR) scores of the groups were

271 similar (Figure 3. and Supplementary Table 1.)

272

273

274

275

276

277

278

279

280

281

282

283

284

285

286

287

\section{Serum cytokines}

The serum cytokine values of the various groups are given in Table 1. The betweengroups baseline (end of week 3), treatment end-point (end of week 7), and study end-point (end of week 11) comparisons showed that the log-transformed serum TNF $\alpha$ rose in GrpP from week 3 to week $7(p=0.049)$ and IL-6 in GrpM Lo fell from week 3 to week $11(p=0.044)$. All the rest of comparisons gave no significant differences (Table 1.).

\section{Cox-2 expression}

The Cox-2 immunostaining values for the study groups are given in Table 2. Cox- 2 expression was undetectable in GrpCo and was very faint in the subchondral bone sub-region of animals in GrpM Hi. In GrpP, low-grade cartilage staining, and a variable, but higher-grade, subchondral bone and bone marrow expression, were observed. Animals from GrpM Lo had no cartilage reactions and only faint Cox-2 staining in subchondral bone and bone marrow (Figure 4.). The Kruskal-Wallis ANOVA test revealed significant differences between GrpP, GrpM Lo and GrpM Hi both for subchondral bone $(\mathrm{p}<0.001)$ and bone marrow $(\mathrm{p}<0.001)$. HolmBonferroni corrected statistics resulted in significantly lower Cox-2 percentages in the subchondral bone in GrpM Hi $(p=0.006, z=3.08, r=0.65)$ and GrpM Lo $(p=0.030, z=2.46$, $\mathrm{r}=0.51$ ) than in GrpP. Cox-2 scores of bone marrow were different in GrpM Hi vs. GrpP $(\mathrm{p}=0.006, \mathrm{z}=3.12, \mathrm{r}=0.66)$, and GrpM Lo vs. GrpP $(\mathrm{p}=0.016, \mathrm{z}=2.67, \mathrm{r}=0.56)$ (Table 2.). 


\section{DISCUSSION}

Different MIA doses have been used previously to provoke chemically osteoarthritis in 293 rats (Guzman et al., 2003; Dumond et al., 2004, Boudenot et al., 2014, Guingamp et al., 1997,

294 Miyamato et al., 2016). However, it is known that beside a rapid and dose-dependent decrease of 295 locomotor activity, a secondary, progressive, long-term loss of spontaneous mobility happens 296 only if high doses of MIA (0.3-3 mg) are applied (Guingamp et al., 1997). Considering this 297 finding, we focused our study on the high-dose MIA (4 mg) induced osteoarthritis.

Data accumulated previously suggest that pharmacokinetics of meloxicam in rats is 299 similar to those in humans, the rat being the preferred species for data extrapolation to humans

300 (Busch et al., 1998). However, there is remarkable pharmacokinetic variability in the results 301 among authors: $\mathrm{t}_{1 / 2}=13 \mathrm{hr}$ in male and $\mathrm{t}_{1 / 2}=37 \mathrm{hr}$ in female rats (Busch et al., 1998); $\mathrm{t}_{1 / 2}=9 \mathrm{hr}$

302 (Aguilar-Mariscal et al., 2007); and $\mathrm{t}_{1 / 2}=19-23$ hrs (Aghazadeh-Habashi \& Jamali, 2008). Our

303 mean value of Cmax obtained $(4.79 \mu \mathrm{g} / \mathrm{mL})$ was higher than the values reported by other

304 authors: $1.1 \mu \mathrm{g} / \mathrm{mL}$ (after a unique dose of $1 \mathrm{mg} / \mathrm{kg}$ (Ochi et al, 2013) or $3.5 \mu \mathrm{g} / \mathrm{mL}$ (after a dose 305 of $0.9 \mathrm{mg} / \mathrm{kg}$ ) (Aghazadeh-Habashi \& Jamali, 2008). The $\mathrm{t}_{1 / 2}$ calculated for non-compartmental 306 pharmacokinetics was similar with those obtained by other groups (Busch et al., 1998;

307 Aghazadeh-Habashi \& Jamali, 2008). The explanation for the high Cmax might be our 308 formulation: meloxicam was dissolved in (50:50) PEG400: $\mathrm{H}_{2} \mathrm{O}$, instead of powder, tablets or 309 aqueous suspension. Considering the long $\mathrm{t}_{1 / 2}$ obtained, we concluded that our meloxicam

310 formulation can be administered once daily, and that the steady-state concentration is probably

311 achieved in about 5 days. The dosage of meloxicam was defined taking into account the human 312 equivalent dose (HED), and the repeated dose toxicity "No Effect Level" (NOEL). 
314 period of 11 weeks post-administration of MIA equals by extrapolation approximately 7 years in

315 humans (Sengupta, 2013). Moreover, since the first inflammatory signs in the MIA model appear

316 at the end of the first week, week 11 can be considered the chronic, late-phase of the MIA-

317 triggered inflammatory response. We hypothesized that low-grade inflammation induced by

318 MIA persists in the subchondral bone and bone marrow in the late-phase reaction of

319 osteoarthritis, thus sustaining cartilage destruction. We also proposed that meloxicam could

320 ameliorate not only the cartilage deterioration, but also the lesions provoked on subchondral

321 bone. To test these hypotheses, we used the OARSI-recommended histological approach in

322 evaluating the osteoarthritis and response to meloxicam treatment.

323 Our major findings were that meloxicam at low-dose $(0.2 \mathrm{mg} / \mathrm{kg})$ and high dose (1

$324 \mathrm{mg} / \mathrm{kg}$ ) had a chondroprotective effect, and the high dose also protected against subchondral

325 bone lesions. The late-phase inflammatory process was alleviated by meloxicam treatment.

326 The presence of a low-grade, local inflammation was highlighted through Cox-2

327 expression that was rarely detectable at the level of cartilage, but was more intense in

328 subchondral bone and bone marrow 11 weeks after the MIA-trigger in the GrpP animals. This

329 low-grade inflammation could be an important clue of the progressive degenerative pathways.

330 Cox-2 expression in subchondral bone and bone marrow was significantly suppressed in GrpM

331 Lo (low-dose meloxicam) and virtually absent in GrpM Hi (high-dose meloxicam) animals, 332 which is evidence of the protection of meloxicam in this model. 
334 offer a more precise evaluation of tissue lesions in osteoarthritis (Pritzker et al., 2006; Gerwin et

335 al., 2010).

We chose four scores of the OARSI histopathology initiative that are relevant not only for

337 the depth and width of cartilage degradation, but also for evaluation of the interacting joint

338 regions: the subchondral bone and the synovial membrane. In our study, the Cartilage

339 Degeneration Score (CDS) and the Total Cartilage Degeneration Width (TCDW) were

340 significantly improved in both MXC-treated rat groups. Both the low and high dose of

341 meloxicam were effective in improving the depth (cartilage degeneration score) and extension

342 (total cartilage degeneration width) of the lesions in the MIA-triggered osteoarthritis. In addition,

343 it is even more important that high-dose meloxicam conferred protection also for the deep

344 cartilage and subchondral bone, since this functional unit might be essential in down-regulation

345 of the persistent inflammation and the long-term degeneration pathway.

346 Application of the subchondral bone damage score in histological assessments may be

347 important in future studies since it is the only simultaneous measure of the deep cartilage-

348 subchondral bone-bone marrow alterations.

Functional models of the joint reveal a complex interplay between cartilage, subchondral

350 bone, and bone marrow. This relationship suggests the possibility of simultaneous investigation

351 of potentially protective drugs both at the levels of cartilage and the subchondral bone (Yuan et

352 al., 2014; Funck-Brentano \& Cohen-Solal, 2011). In patients of the Multicenter Osteoarthritis

353 study, bone-marrow changes in osteoarthritis were tightly associated with subchondral bone

354 attrition (Roemer et al., 2010). The time-sequence of histological lesions generated by MIA until 
355 day 56, post-administration has been documented (Guzman et al., 2003). Cartilage degradation,

356 fibrillation and chondrocyte degeneration are early phenomena, followed by the involvement of

357 subchondral bone that becomes obvious at the end of the first week after the injection and lasts

358 for at least 56 days (Guzman et al., 2003; Morenko et al., 2004; Pitcher, Sousa-Valente \&

359 Malcangio, 2016). The most remarkable consequences seen are the intensification of bone

360 remodelling and the appearance of microfractures and osteophytes, but mesenchymal

361 transformation of bone marrow spaces subjacent to the most affected cartilage zones also is

362 present. MIA also causes a reduction of bone mineral density in the proximal tibia (Boudenot et

363 al., 2014). Histologically, many features of the late-phase lesions of MIA-induced osteoarthritis

364 resemble those of the advanced-phase human disease (Lorenz \& Richter, 2006).

365 Meloxicam has a high anti-inflammatory potential because of its preferential Cox-2

366 inhibition, but its chondroprotective effect is not unanimously supported by literature reports.

367 Early studies showed that meloxicam is chondroneutral and does not influence proteoglycan

368 synthesis (Engelhardt, 1996). However, in cell-culture studies, meloxicam generated favourable

369 effects on overall proteoglycan synthesis (Blot et al., 2000). In an animal study (Jones et al.,

370 2010), $3 \mathrm{mg} / \mathrm{kg}$ meloxicam in monotherapy did not improve cartilage lesions, but it correlated

371 with a higher bone-volume percentage in female Sprague-Dawley rats that underwent knee triad

372 injury. In contrast with these findings, Wen et al. (2013) recently elucidated in anterior crucial

373 ligament trans-section-induced osteoarthritis in Wistar rats a significant improvement of the

374 OARSI and synovial scores with doses of $0.25 \mathrm{mg} / \mathrm{kg}$ and $1 \mathrm{mg} / \mathrm{kg}$ meloxicam (Wen et al., 375 2013).

Other Cox-2 selective inhibitors, such as celecoxib, improved proteoglycan synthesis and

377 turnover and synovial release of IL-1 $\beta$ and TNF $\alpha$ in samples from human patients who 
378 underwent knee-replacement surgery (de Boer et al., 2009). Celecoxib in combination with

379 rebamipide is highly efficient in reducing osteoclast number in the subchondral bone marrow in

380 MIA-induced rat osteoarthritis (Moon et al., 2013).

The mechanisms of disease progression in the MIA-induced osteoarthritis are not well-

382 known; however, the time-specific participation of pro-inflammatory molecules in cartilage-bone

383 crosstalk has been documented (Funck-Brentano \&Cohen-Solal, 2011). Possibly, the first

384 inflammatory burst after MIA injection, and then a continuing low-grade inflammation nested in

385 the subchondral bone and bone marrow, contribute to the catabolic reprogramming of

386 chondrocytes and to the emergence of inflammatory transcriptional "go-signals" (Liu-Bryan

387 \&Terkeltaub, 2015). This phenomenon could be an explanation for the overall improvement of

388 cartilage and subchondral bone-damage by the high-dose meloxicam in our experiments. Bone

389 marrow stimulation followed by implantation of acellular biomaterials significantly improved

390 cartilage repair in several preclinical studies (Pot et al., 2016).

391 Much evidence supports the pathogenic role of pro-inflammatory (IL-1, IL-6, TNF $\alpha$, IL-

392 15, IL-17) and anti-inflammatory (IL-4, IL-10) cytokines in osteoarthritis (Kapoor et al., 2011;

393 Wojdasiewicz et al., 2014); however, the presence of a systemic inflammation in osteoarthritis

394 has not been confirmed unanimously. In the cartilage, TNF $\alpha$ causes a focal tissue loss due to the

395 fact that chondrocytes wear variable amounts of p75 TNF $\alpha$ receptor (Westacott et al., 2000).

396 Local chemokines, such as CCL20 can induce IL-6 and Cox-2 in explanted donor and

397 osteoarthritic chondrocytes (Alaaeddine et al., 2015). Increased circulating levels of IL-6 and IL-

39810 have been reported in painful osteoarthritis (Imamura et al., 2015), but this reaction might be

399 absent in the late-disease phase. Isolated mechanical injuries of the joint may lead to self-

400 perpetuating, chronic local inflammatory reactions (Sokolove et al., 2013). In our placebo-group, 
401 the levels of serum TNF $\alpha$ ascended from week 3 to week 7. The source of this systemic

402 elevation might have been the progressive cartilage destruction and erosion. Meloxicam did not

403 influence the IL-10 and the TNF $\alpha$ levels neither in GrpM Lo nor in GrpM Hi. In parallel, IL-6 at

404 GrpM Lo fell about 40\% from week 3 to week 11, but this effect was not seen with GrpM Hi.

405 The decrease of IL-6 could bear a therapeutic benefit in GrpM Lo; however, it was not

406 characteristic of the high-dose meloxicam with a more pronounced histological effect. A

407 significant drawback for interpretation of these data is the source of cytokines used in our study:

408 the synovial fluid instead of serum probably would be more useful in establishing the dose-

409 related local effects. However, comparison of cytokine profiles of the synovial fluid and the

410 cartilage proved difference (Tsuchida et al., 2014).

There are different opinions in the literature regarding the applicability of MIA-models to

412 study of the spontaneous progression of osteoarthritis. According to some authors, the

413 consequences of MIA injection imitate an inflammatory arthritis rather than spontaneous disease

414 (Teeple et al., 2013). The transcriptional profile overlap between human and MIA-induced rat

415 osteoarthritis is poor, but the comparison was made only for cartilage (Teeple et al., 2013);

416 others studies, in contrast, emphasize that the MIA-model is minimally invasive, rapid, and

417 reproducible, with comparable degenerative and histological changes to the human anterior

418 cruciate ligament-induced osteoarthritis (Thysen, Luyten \&Lories, 2015; Naveen et al., 2014).

419 Our data bring evidence that both low dose $(0.2 \mathrm{mg} / \mathrm{kg})$ and high dose $(1 \mathrm{mg} / \mathrm{kg})$

420 meloxicam are efficient alternatives, with reference both to the depth (CDS, SBD) and extension

421 (TCDW) of the lesions in MIA-triggered osteoarthritis. In addition, it is even more important that 422 high-dose meloxicam conferred protection also for the deep cartilage and subchondral bone, 
423 since this functional unit might be essential in down-regulation of the persistent inflammation

424 and the long-term degeneration pathway.

425

\section{CONCLUSIONS}

426

In this rat model of MIA-induced late-phase osteoarthritis, both low-dose and high-dose

427 meloxicam had a chondroprotective effect, and the high dose also protected against subchondral

428 bone lesions. The importance of this finding lies in the assumption that subchondral bone and

429 bone marrow alterations sustain and perpetuate the deterioration of cartilage. Although findings

430 in the rat model cannot be directly extrapolated to human disease, the favourable changes seen at

431 the level of subchondral bone and bone marrow, suggest that meloxicam therapy, especially in

432 high doses, can attenuate chronic-phase disease progression.

433 More evidence, including immunohistochemical characterization of the inflammatory

434 infiltrate and fibroblastic transformation, is needed to highlight the specific action of meloxicam 435 on subchondral bone and bone marrow.

\section{Acknowledgements}

We thank Professor Daniela-Lucia Muntean for the organizational support, Dr.Gabriela

Marcus, Ana Popeiu and Teodora Popeiu for their participation in animal treatment and

440 surveillance, and Dr. Miklós Bob for the kind interpretation of radiological data. 
444

445

446

447

448

449

450

451

452

453

454

455

456

457

458

459

460

461

462

463

464

465

466

467

468

469

470

471

472

473

474

475

476
Aghazadeh-Habashi A, and Jamali F. 2008. Pharmacokinetics of meloxicam administered as regular and fast dissolving formulations to the rat: Influence of gastrointestinal dysfunction on the relative bioavailability of two formulations. European Journal of Pharmaceutics and Biopharmaceutics 70:889-894. 10.1016/j.ejpb.2008.07.013

Aguilar-Mariscal H, Patino-Camacho SI, Rodriguez-Silverio J, Torres-Lopez JE, and Flores-Murrieta FJ. 2007. Oral pharmacokinetics of meloxicam in the rat using a highperformance liquid chromatography method in micro-whole-blood samples. Methods and Findings in Experimental and Clinical Pharmacology 29:587-591. 10.1358/mf.2007.29.9.1116314

Alaaeddine N, Antoniou J, Moussa M, Hilal G, Kreichaty G, Ghanem I, Abouchedid W, Saghbini E, and Di Battista JA. 2015. The chemokine CCL20 induces proinflammatory and matrix degradative responses in cartilage. Inflammation Research 64:721-731. 10.1007/s00011015-0854-5

Altman R, Hochberg M, Gibofsky A, Jaros M, and Young C. 2015. Efficacy and safety of low-dose SoluMatrix meloxicam in the treatment of osteoarthritis pain: a 12-week, phase 3 study. Current Medical Research and Opinion 31:2331-2343. 10.1185/03007995.2015.1112772

Bendele AM. 2001. Animal models of osteoarthritis. Journal of musculoskeletal \& neuronal interactions 1:363-376.

Berenbaum F. 2013. Osteoarthritis as an inflammatory disease (osteoarthritis is not osteoarthrosis!). Osteoarthritis and Cartilage 21:16-21. 10.1016/j.joca.2012.11.012

Blot L, Marcelis A, Devogelaer JP, and Manicourt DH. 2000. Effects of diclofenac, aceclofenac and meloxicam on the metabolism of proteoglycans and hyaluronan in osteoarthritic human cartilage. British Journal of Pharmacology 131:1413-1421. 10.1038/sj.bjp.0703710

Boudenot A, Presle N, Uzbekov R, Toumi H, Pallu S, and Lespessailles E. 2014. Effect of interval-training exercise on subchondral bone in a chemically-induced osteoarthritis model. Osteoarthritis and Cartilage 22:1176-1185. 10.1016/j.joca.2014.05.020

Busch U, Schmid J, Heinzel G, Schmaus H, Baierl J, Huber C, and Roth W. 1998. Pharmacokinetics of meloxicam in animals and the relevance to humans. Drug Metabolism and Disposition 26:576-584.

Custers RJH, Creemers LB, Verbout AJ, van Rijen MHP, Dhert WJA, and Saris DBF. 2007. Reliability, reproducibility and variability of the traditional Histologic/Histochemical Grading System vs the new OARSI Osteoarthritis Cartilage Histopathology Assessment System. Osteoarthritis and Cartilage 15:1241-1248. 10.1016/j.joca.2007.04.017 
477

478

479

480

481

482

483

484

485

486

487

488

489

490

491

492

493

494

495

496

497

498

499

500

501

502

503

504

505

506

507

508

509

510

511

512

de Boer TN, Huisman AM, Polak AA, Niehoff AG, van Rinsum AC, Saris D, Bijlsma JWJ, Lafeber FJPG, and Mastbergen SC. 2009. The chondroprotective effect of selective COX-2 inhibition in osteoarthritis: ex vivo evaluation of human cartilage tissue after in vivo treatment. Osteoarthritis and Cartilage 17:482-488. 10.1016/j.joca.2008.09.002

Dumond H, Presle N, Pottie P, Pacquelet S, Terlain B, Netter P, Gepstein A, Livne E, and Jouzeau JY. 2004. Site specific changes in gene expression and cartilage metabolism during early experimental osteoarthritis. Osteoarthritis and Cartilage 12:284-295. 10.1016/j.joca.2003.11.008

Engelhardt G. 1996. Pharmacology of meloxicam, a new non-steroidal anti-inflammatory drug with an improved safety profile through preferential inhibition of COX-2. British Journal of Rheumatology 35:4-12.

Funck-Brentano T, and Cohen-Solal M. 2011. Crosstalk between cartilage and bone: When bone cytokines matter. Cytokine \& Growth Factor Reviews 22:91-97. 10.1016/j.cytogfr.2011.04.003

Gerwin N, Bendele A, Glasson S, and Carlson C. 2010. The OARSI histopathology initiative - recommendations for histological assessments of osteoarthritis in the rat. Osteoarthritis and Cartilage 18:S24-S34. 10.1016/j.joca.2010.05.030

Guingamp C, GegoutPottie P, Philippe L, Terlain B, Netter P, and Gillet P. 1997. Monoiodoacetate-induced experimental osteoarthritis - A dose-response study of loss of mobility, morphology, and biochemistry. Arthritis and Rheumatism 40:1670-1679. 10.1002/art.1780400917

Guzman RE, Evans MG, Bove S, Morenko B, and Kilgore K. 2003. Mono-iodoacetateinduced histologic changes in subchondral bone and articular cartilage of rat femorotibial joints: An animal model of osteoarthritis. Toxicologic Pathology 31:619-624.

$10.1080 / 01926230390241800$

Imamura M, Ezquerro F, Marcon Alfieri F, Vilas Boas L, Tozetto-Mendoza TR, Chen J, Ozcakar L, Arendt-Nielsen L, and Rizzo Battistella L. 2015. Serum levels of proinflammatory cytokines in painful knee osteoarthritis and sensitization. International journal of inflammation 2015:329792-329792. 10.1155/2015/329792

Jones MD, Tran CW, Li G, Maksymowych WP, Zernicke RF, and Doschak MR. 2010. In Vivo Microfocal Computed Tomography and Micro-Magnetic Resonance Imaging Evaluation of Antiresorptive and Antiinflammatory Drugs as Preventive Treatments of Osteoarthritis in the Rat. Arthritis and Rheumatism 62:2726-2735. 10.1002/art.27595

Kapoor M, Martel-Pelletier J, Lajeunesse D, Pelletier J-P, and Fahmi H. 2011. Role of proinflammatory cytokines in the pathophysiology of osteoarthritis. Nature Reviews Rheumatology 7:33-42. 10.1038/nrrheum.2010.196 
513

514

515

516

517

518

519

520

521

522

523

524

525

526

527

528

529

530

531

532

533

534

535

536

537

538

539

540

541

542

543

544

545

546

547

548

549

Kellgren JH, and Lawrence JS. 1957. Radiological assessment of osteo-arthrosis. Ann Rheum Dis 16: 494-502.

Kilkenny C, Browne WJ, Cuthill IC, Emerson M, and Altman DG. 2010. Improving Bioscience Research Reporting: The ARRIVE Guidelines for Reporting Animal Research. Plos Biology 8. 10.1371/journal.pbio. 1000412

Liu-Bryan R, and Terkeltaub R. 2015. Emerging regulators of the inflammatory process in osteoarthritis. Nature Reviews Rheumatology 11:35-44. 10.1038/nrrheum.2014.162

Lorenz H, and Richter W. 2006. Osteoarthritis: Cellular and molecular changes in degenerating cartilage. Progress in Histochemistry and Cytochemistry 40:135-163. 10.1016/j.proghi.2006.02.003

McAlindon TE, Bannuru RR, Sullivan MC, Arden NK, Berenbaum F, Bierma-Zeinstra SM, Hawker GA, Henrotin Y, Hunter DJ, Kawaguchi H, Kwoh K, Lohmander S, Rannou F, Roos EM, and Underwood M. 2015. 2014 OARSI Guidelines for the Non-Surgical Management of Knee Osteoarthritis (vol 22, pg 363, 2014). Osteoarthritis and Cartilage 23:1026-1034. 10.1016/j.joca.2015.02.014

Miyamoto S, Nakamura J, Ohtori S, Orita S, Omae T, Nakajima T, Suzuki T, and Takahashi K. 2016. Intra-articular injection of mono-iodoacetate induces osteoarthritis of the hip in rats. Bmc Musculoskeletal Disorders 17. 10.1186/s12891-016-0985-Z

Moon S-J, Park J-S, Jeong J-H, Yang E-J, Park M-K, Kim E-K, Park S-H, Kim H-Y, Cho M-L, and Min J-K. 2013. Augmented chondroprotective effect of coadministration of celecoxib and rebamipide in the monosodium iodoacetate rat model of osteoarthritis. Archives of Pharmacal Research 36:116-124. 10.1007/s12272-013-0010-0

Morenko BJ, Bove SE, Chen LG, Guzman RE, Juneau P, Bocan TMA, Peter GK, Arora $\mathrm{R}$, and Kilgore KS. 2004. In vivo micro computed tomography of subchondral bone in the rat after intra-articular administration of monosodium iodoacetate. Contemporary Topics in Laboratory Animal Science 43:39-43.

Musumeci G, Aiello FC, Szychlinska MA, Di Rosa M, Castrogiovanni P, and Mobasheri A. 2015. Osteoarthritis in the XXIst Century: Risk Factors and Behaviours that Influence Disease Onset and Progression. International Journal of Molecular Sciences 16:6093-6112. 10.3390/ijms16036093

Nair AB, and Jacob S. 2016. A simple practice guide for dose conversion between animals and human. J Basic Clin Pharma 7(2):27-31. doi: 10.4103/0976-0105.177703.

Naveen SV, Ahmad RE, Hui WJ, Suhaeb AM, Murali MR, Shanmugam R, and Kamarul T. 2014. Histology, Glycosaminoglycan Level and Cartilage Stiffness in MonoiodoacetateInduced Osteoarthritis: Comparative Analysis with Anterior Cruciate Ligament Transection in Rat Model and Human Osteoarthritis. International Journal of Medical Sciences 11:97-105. 10.7150/ijms.6964 
550

551

552

553

554

555

556

557

558

559

560

561

562

563

564

565

566

567

568

569

570

571

572

573

574

575

576

577

578

579

580

581

582

583

584
Ochi M, Inoue R, Yamauchi Y, Yamada S, and Onoue S. 2013. Development of Meloxicam Salts with Improved Dissolution and Pharmacokinetic Behaviors in Rats with Impaired Gastric Motility. Pharmaceutical Research 30:377-386. 10.1007/s11095-012-0878-2

Pitcher T, Sousa-Valente J, and Malcangio M. 2016. The Monoiodoacetate Model of Osteoarthritis Pain in the Mouse. Jove-Journal of Visualized Experiments. 10.3791/53746

Pot MW, Gonzales VK, Buma P, IntHout J, van Kuppevelt TH, de Vries RBM, and Daamen WF. 2016. Improved cartilage regeneration by implantation of acellular biomaterials after bone marrow stimulation: a systematic review and meta-analysis of animal studies. PeerJ 4:e2243; DOI 10.7717/peerj.2243

Pritzker KPH, Gay S, Jimenez SA, Ostergaard K, Pelletier JP, Revell PA, Salter D, and van den Berg WB. 2006. Osteoarthritis cartilage histopathology: grading and staging. Osteoarthritis and Cartilage 14:13-29. 10.1016/j.joca.2005.07.014

Roemer FW, Neogi T, Nevitt MC, Felson DT, Zhu Y, Zhang Y, Lynch JA, Javaid MK, Crema MD, Torner J, Lewis CE, and Guermazi A. 2010. Subchondral bone marrow lesions are highly associated with, and predict subchondral bone attrition longitudinally: the MOST study. Osteoarthritis and Cartilage 18:47-53. 10.1016/j.joca.2009.08.018

Sengupta P. 2013. The Laboratory Rat: Relating Its Age With Human's. International journal of preventive medicine 4:624-630.

Sokolove J, and Lepus CM. 2013. Role of inflammation in the pathogenesis of osteoarthritis: latest findings and interpretations. Therapeutic advances in musculoskeletal disease 5:77-94. 10.1177/1759720x12467868

Teeple E, Jay G, Elsaid K, and Fleming B. 2013. Animal Models of Osteoarthritis: Challenges of Model Selection and Analysis. Aaps Journal 15:438-446. 10.1208/s12248-0139454-x

Thysen S, Luyten FP, and Lories RJU. 2015. Targets, models and challenges in osteoarthritis research. Disease Models \& Mechanisms 8:17-30. 10.1242/dmm.016881

Tsuchida AI, Beekhuizen M, t Hart MC, Radstake T, Dhert WJA, Saris DBF, van Osch G, and Creemers LB. 2014. Cytokine profiles in the joint depend on pathology, but are different between synovial fluid, cartilage tissue and cultured chondrocytes. Arthritis Research \& Therapy 16. 10.1186/s13075-014-0441-0

Wen ZH, Tang CC, Chang YC, Huang SY, Chen CH, Wu SC, Hsieh SP, Hsieh CS, Wang KY, Lin SY, Lee HL, Lee CH, Kuo HC, Chen WF, and Jean YH. 2013. Intra-articular injection of the selective cyclooxygenase-2 inhibitor meloxicam (Mobic) reduces experimental osteoarthritis and nociception in rats. Osteoarthritis and Cartilage 21:1976-1986.

10.1016/j.joca.2013.09.005 
585 Yuan X, Meng H, Wang Y, Peng J, Guo Q, Wang A, and Lu S. 2014. Bone-cartilage 586 interface crosstalk in osteoarthritis: potential pathways and future therapeutic strategies.

587 Osteoarthritis and Cartilage 22:1077-1089. 10.1016/j.joca.2014.05.023

588 Yu D, Xu J, Liu F, Wang X, Mao Y, and Zhu Z. 2016. Subchondral bone changes and the 589 impacts on joint pain and articular cartilage degeneration in osteoarthritis. Clin. Exp. Rheumatol. 590 Aug 31. [Epub ahead of print]

591 Westacott CI, Barakat AF, Wood L, Perry MJ, Neison P, Bisbinas I, Armstrong L, Millar $592 \mathrm{AB}$, and Elson CJ. 2000. Tumor necrosis factor alpha can contribute to focal loss of cartilage in 593 osteoarthritis. Osteoarthritis and Cartilage 8:213-221. 10.1053/joca.1999.0292

594 Westacott CI, Webb GR, Warnock MG, Sims JV, and Elson CJ. 1997. Alteration of 595 cartilage metabolism by cells from osteoarthritic bone. Arthritis and Rheumatism 40:1282-1291. 596 10.1002/1529-0131(199707)40:7<1282::aid-art13>3.0.co;2-e

597 Wojdasiewicz P, Poniatowski LA, and Szukiewicz D. 2014. The Role of Inflammatory 598 and Anti-Inflammatory Cytokines in the Pathogenesis of Osteoarthritis. Mediators of 599 Inflammation. 10.1155/2014/561459

600 


\section{Figure 1 (on next page)}

Flowchart representation of the experimental design and timeline.

OA, osteoarthritis; K-L grade, Kellgren-Lawrence grade; MXC LD, meloxicam, low-dose; MXC HD, meloxicam, high-dose. 


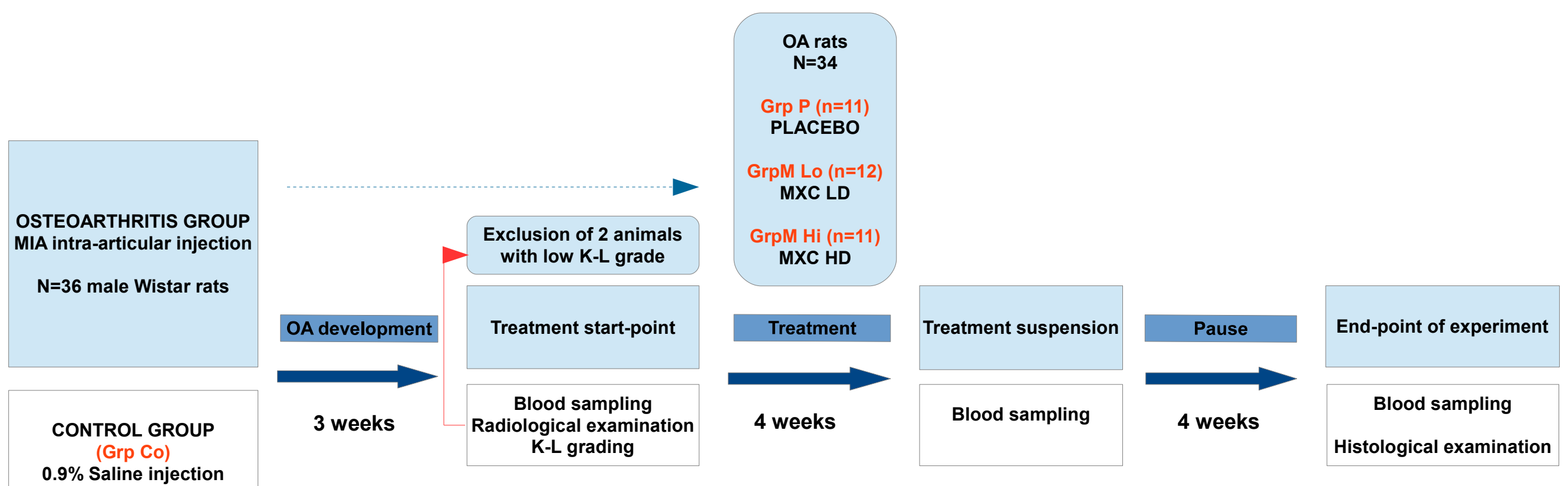




\section{Figure 2}

Histological findings in the studied groups.

2 a-c. MIA-induced joint tissue lesions in Grp P. a. H\&E stain, 10x: deformation of the articular surface, cartilage denudation with microfractures (arrow), bone remodeling, and mesenchymal transformation of the bone marrow (arrowhead). b. H\&E stain, 20x: elongated and flattened chondrocytes and extensive zones lacking viable cells (arrow). c. PAS stain, 10x: superficial zone of cartilage with loss of matrix in the upper one-third (arrow). $\mathbf{2} \mathbf{d}$-f.

Cartilage and subchondral bone lesions in GrpM Lo. d. H\&E stain, 20x: intact superficial zone with edema and deep fibrillation, disorientation and flattening of the chondrocytes. e. H\&E stain, 10x: erosion with cartilage matrix loss, branched fissure (arrow). f. H\&E stain, 10x: cartilage erosion; vertical, branched fissures (arrow), cysts (arrowhead) and mesenchymal changes affecting up to three-fourths of the bone marrow volume. $\mathbf{2} \mathbf{~ g - i . ~}$ Cartilage and subchondral bone lesions in GrpM Hi. g. PAS stain, 10x: intact superficial zone, edema, focal matrix condensation (arrow). h. H\&E stain, 10x: disorientation of chondron columns, with cell death, cell clustering and hypertrophy. Bone marrow mesenchymal changes involving approximately one-fourth of the total volume and increased thickening of the subchondral bone marrow. i. H\&E stain, 10x: intact surface with cell death and hypertrophy in the superficial zone, matrix edema, and no marrow changes in the subchondral bone. 


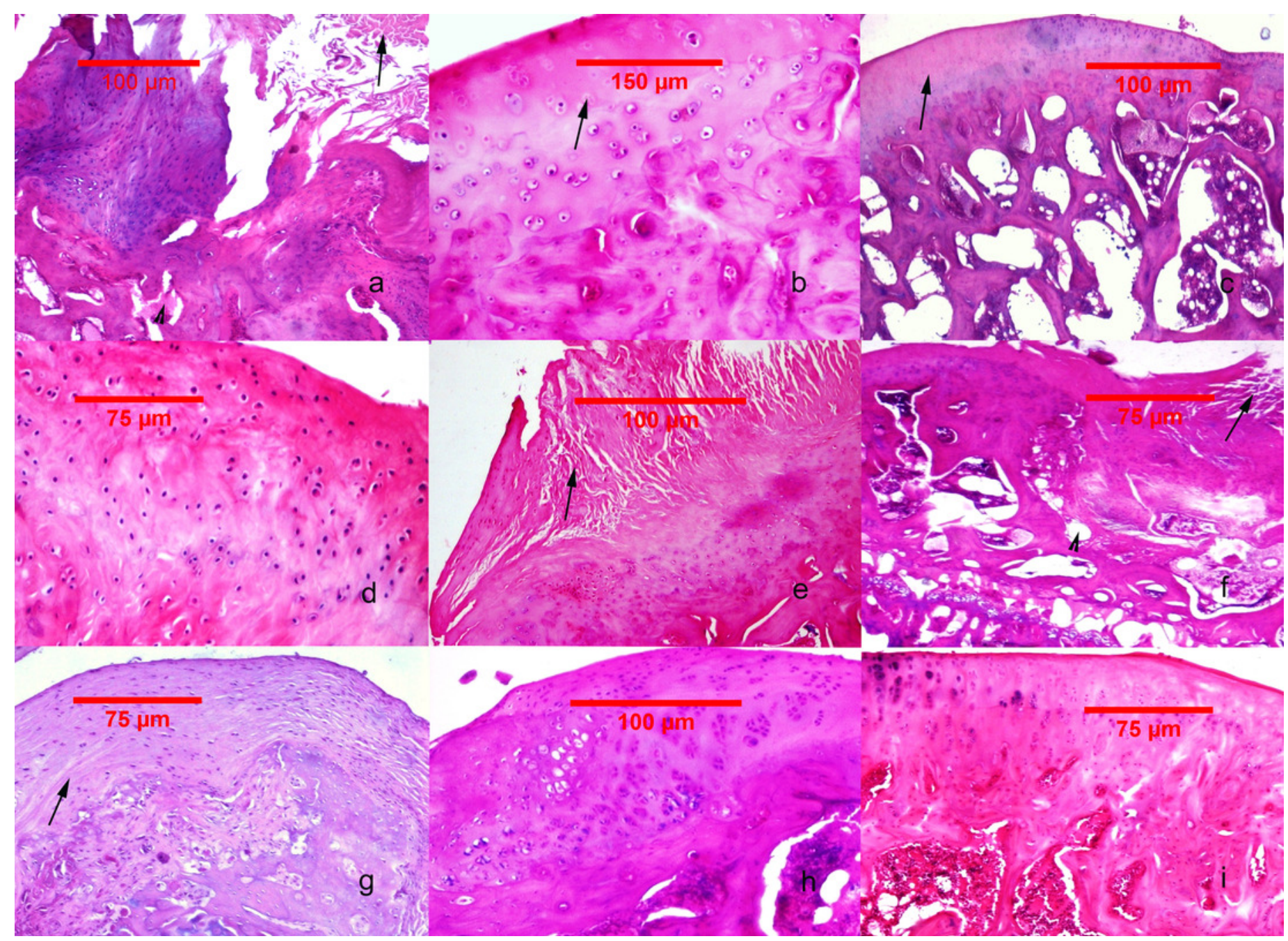


Figure 3 (on next page)

Scatter plot representation of the histological scores.

A - CDS, cartilage degeneration score, B - TCDW, total cartilage degeneration width, C- SBD, calcified cartilage and subchondral bone damage score, D - SR, synovial reaction. Values shown as median and interquartile range. Significant differences marked with * for $p<0.05$, ** $p<0.01$. Comparisons for GrpCo shown in boxes, in order for GrpP, GrpM Lo, GrpM Hi. 
PeerJ
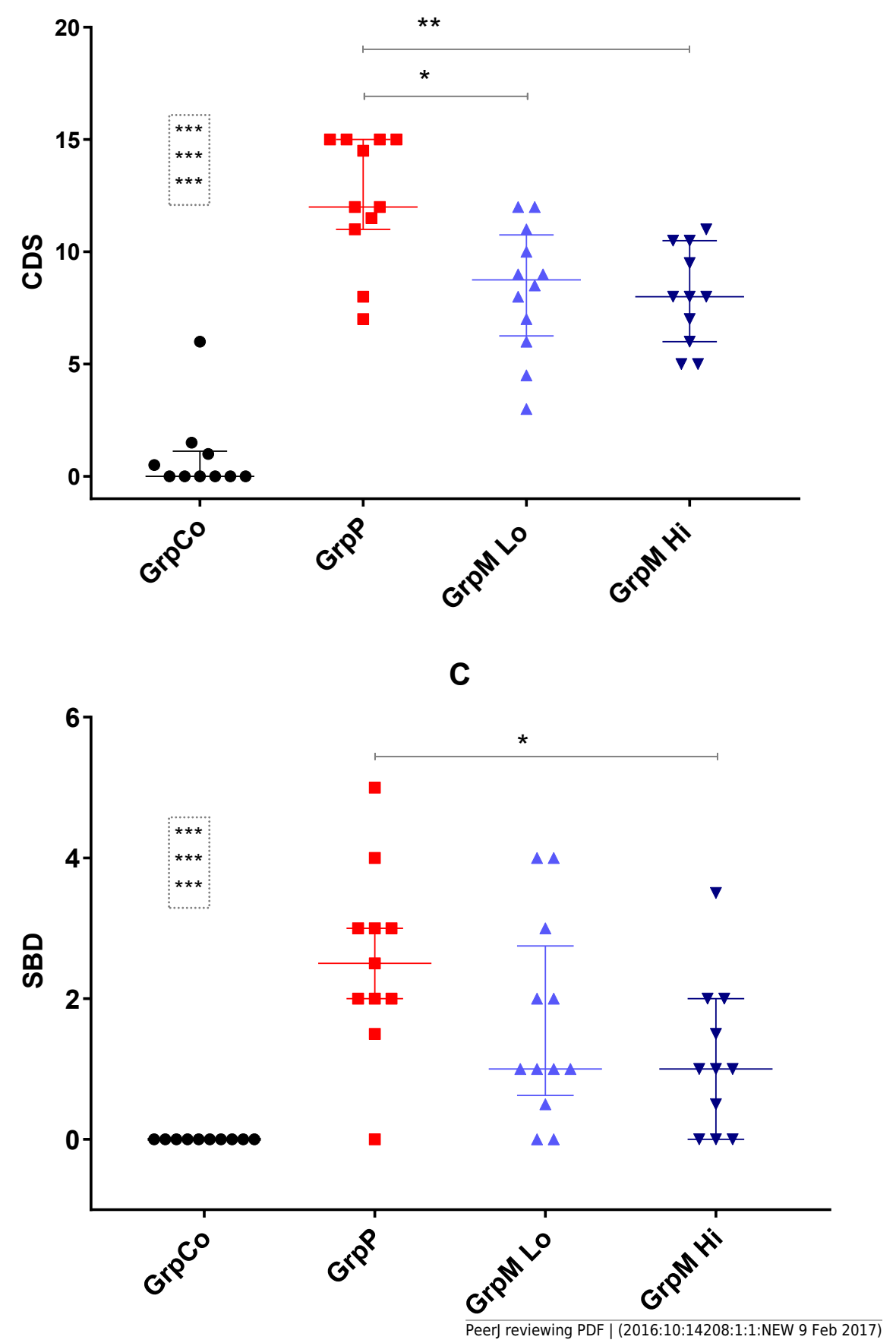

Manuscript to be reviewed ${ }_{\mathbf{B}}$
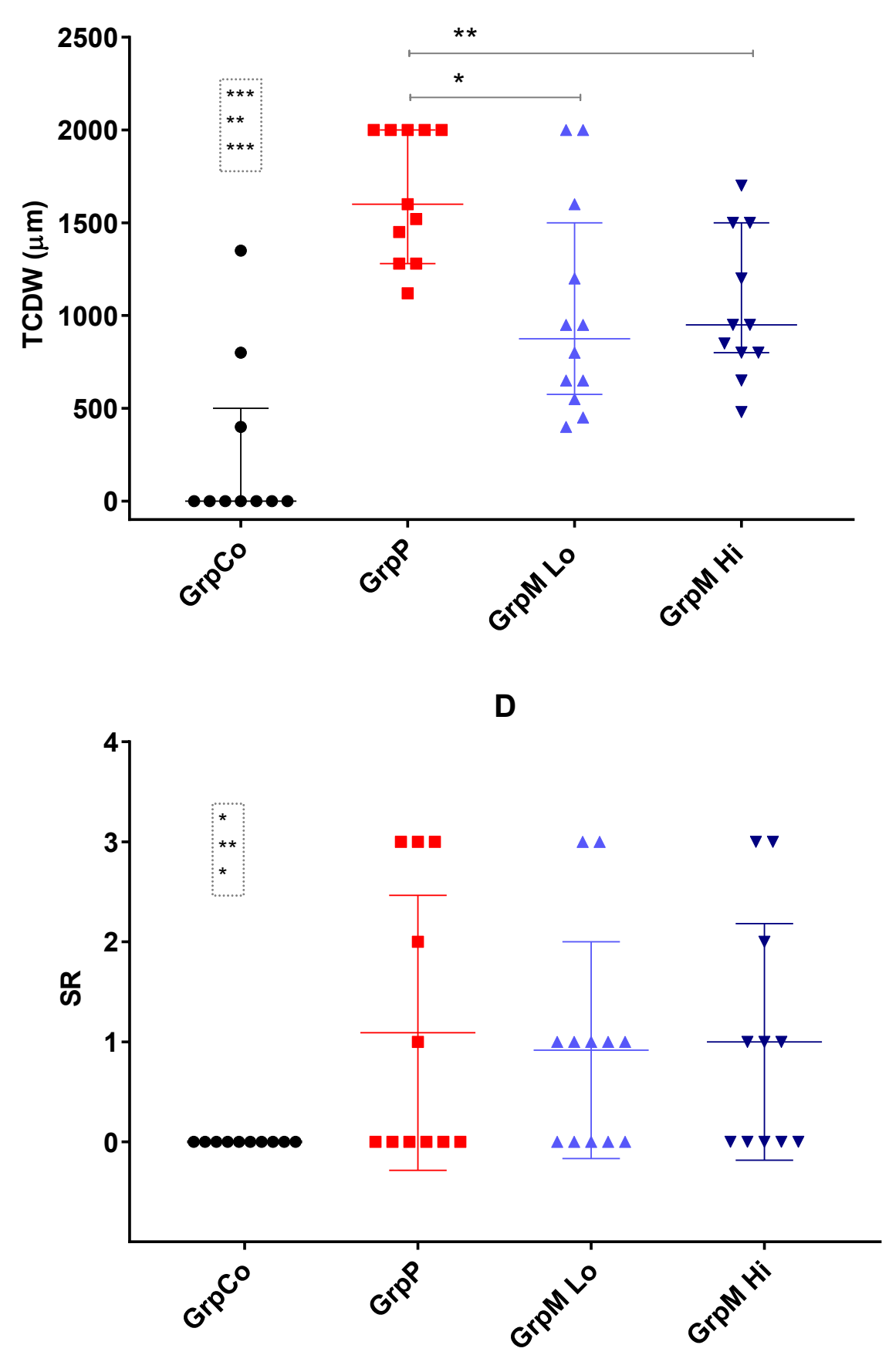


\section{Figure 4}

Cox-2 immunohistochemistry staining of the joint tissues.

a. Animal from Grp P: bone marrow with positive staining, showing focal cytoplasmic and membrane reaction in mononuclear cells (arrow). b. Animal from GrpM Lo: cartilage-bone transitional zone with a few Cox-2 positive fibroblasts, fibrocytes, and mononuclear cells (arrows). c. Animal from GrpM Hi: hypercellular bone marrow, with rare Cox-2-positive cells (arrows).

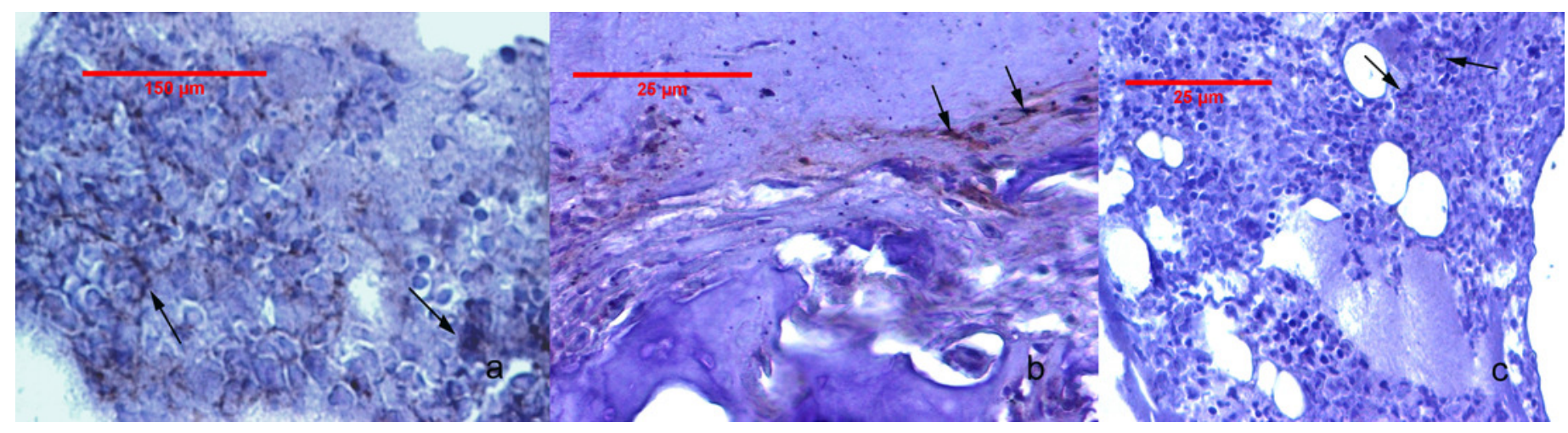




\section{Table $\mathbf{1}$ (on next page)}

The serum cytokine values in the study groups.

Values expressed as mean \pm SE (standard error). w3- end of week 3 (baseline), w7-end of week 7, w11- end of week 11. $* p<0.05$, shown for paired comparisons of log-transformed IL-6 values in GrpM Lo, ${ }^{\dagger} p<0.05$, shown for paired comparisons of log-transformed TNF values in GrpP. 


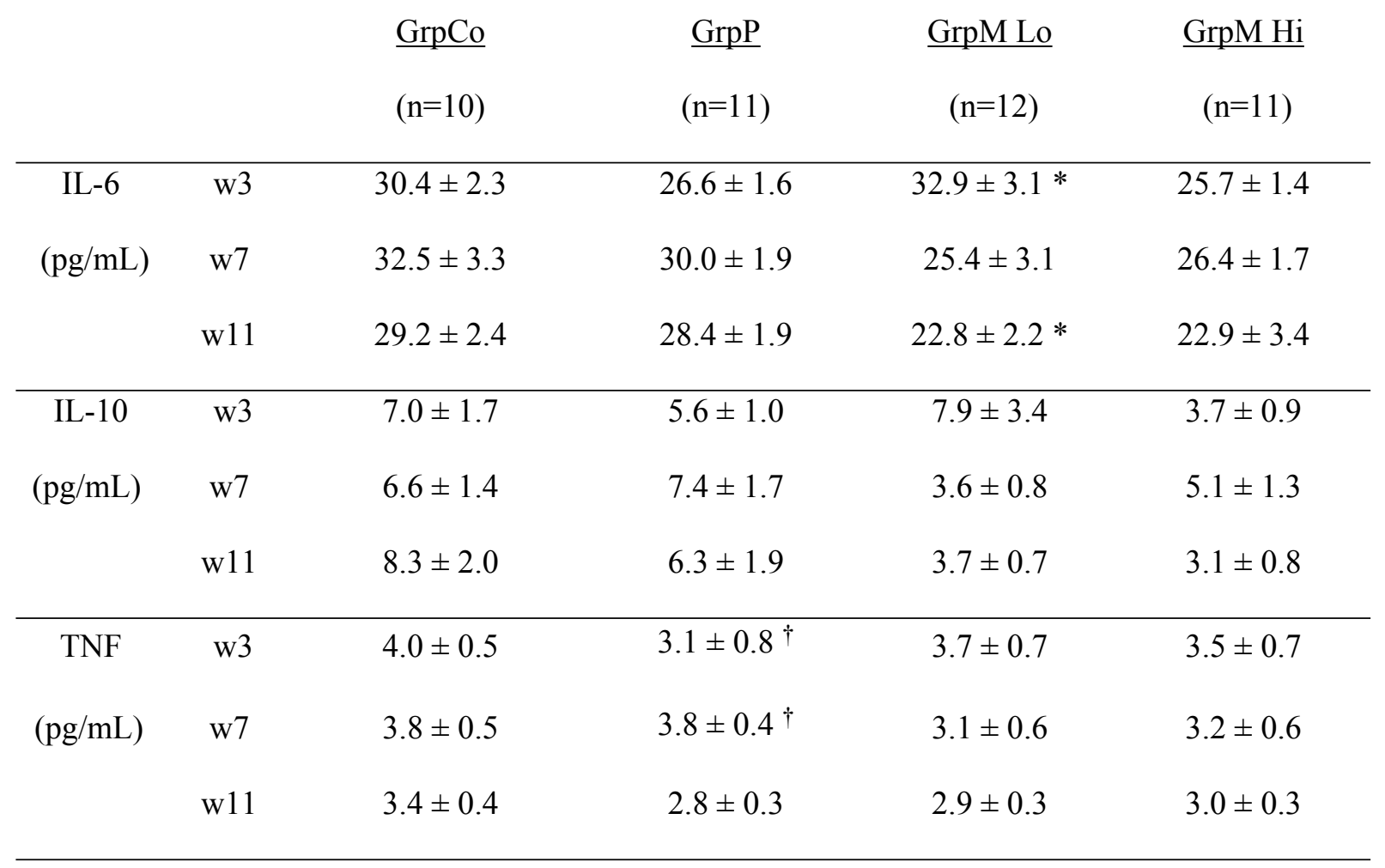

1 


\section{Table 2 (on next page)}

Cox-2 immunostaining scores of the studied groups.

Cox-2 staining expressed as percentage values, expressed as mean \pm SE (standard error). Holm-Bonferroni adjusted ${ }^{*} p<0.05,{ }^{+\dagger} p<0.01$ shown for paired comparisons between Grp P and GrpM Lo (marked with*), and GrpP and GrpM Hi (marked with ${ }^{\dagger}$ ), respectively. Significance values for comparisons of GrpCo are not shown. 


\begin{tabular}{|c|c|c|c|c|}
\hline Cox-2 staining & $\underline{\mathrm{GrpCo}}$ & GrpP & $\underline{\text { GrpM Lo }}$ & $\underline{\text { GrpM Hi }}$ \\
\hline score & $(n=10)$ & $(n=11)$ & $(n=12)$ & $(n=11)$ \\
\hline cartilage & 0 & $1.82 \pm 0.66$ & 0 & 0 \\
\hline subchondral bone & 0 & $6.54 \pm 1.60 *, \dagger \dagger$ & $1.83 \pm 0.58 *$ & $0.81 \pm 0.55 \dagger$ \\
\hline bone marrow & 0 & $12.18 \pm 3.74 *, \dagger$ & $1.58 \pm 0.58 *$ & $0.73 \pm 0.48 \dagger$ \\
\hline
\end{tabular}

1 